\title{
Thienopyridine Antiplatelet Agent
}

National Cancer Institute

\section{Source}

National Cancer Institute. Thienopyridine Antiplatelet Agent. NCI Thesaurus. Code C80483.

A class of agents that target at platelet ADP receptor or purinergic receptor P2RY12 with antiplatelet activity. 